\title{
DESIGN AND DEVELOPMENT OF ELECTRO-OPTICAL SYSTEM FOR ACQUISITION OF PPG SIGNALS FOR THE ASSESSMENT OF CARDIOVASCULAR SYSTEM
}

\author{
Jayadevappa B.M1, Kiran Kumar G.H², Anjaneya L.H ${ }^{3}$, Mallikarjun S. Holi ${ }^{4}$ \\ ${ }^{\text {I}}$ Professor and Head, Electronics and Instrumentation Engineering, Bapuji Institute of Engineering and Technology, \\ Davangere, Karnataka, India \\ ${ }^{2}$ Assistant Professor, Electronics and Communication Engineering, Bapuji Institute of Engineering and Technology, \\ Davangere, Karnataka, India \\ ${ }^{3}$ Associate Professor, Biomedical Engineering, Bapuji Institute of Engineering and Technology, Davangere, \\ Karnataka, India \\ ${ }^{4}$ Professor and Chairman, Electronics and Instrumentation Engineering, University B.D.T. College of Engineering, \\ Davangere, Karnataka, India
}

\begin{abstract}
The revolution in semiconductor technology has lead to the development of efficient optical techniques for acquisition of biomedical signals which can be used effectively in diagnostics. The interaction of light with biological tissue is complex and includes the optical processes like scattering, absorption, reflection, transmission and fluorescence. The amount of light received by the photo-detector varies in accordance with the blood volume, blood vessel wall movement and the orientation of red blood cells $(R B C)$. In the present work a real-time electro-optical based photoplethysmograpgh system is developed to monitor and record the variations in the volume of blood with each heart beat resulting in the form of a signal called photoplethysmogram $(P P G)$. The developed sensor unit consists of a source of light and photo-detector both packed in the form of probe and fitted to patient's index finger. The PPG signal acquired comprise a pulsatile (AC) waveform attributed to cardiac synchronous changes in the blood volume with each heart beat and is superimposed on a slowly varying $(D C)$ baseline with various lower frequency components attributed to respiration, sympathetic nervous system activity and thermoregulation. For remote monitoring of PPG signals, a system is designed and developed based on ZigBee wireless technology. From the transmitted and received PPG signals, parameters like pulse rate $(P R)$, average pulse rate $(A P R)$, pulse rate variability $(P R V)$ and frequency spectrum can be determined which are of diagnostic importance. The PPG system designed and developed in the present work is non-invasive, portable, reliable and cost effective. The system can be effectively used for handicapped patients, ambulatory conditions and in post operative care units for continuous monitoring and assessment of cardiovascular patients. The preliminary study and results shows that the developed wireless PPG system can be an alternative to the traditional methods used for remote monitor of cardiovascular and respiratory system and their disorders.
\end{abstract}

Keywords: Photoplethysmogram (PPG), cardiovascular system, pulse rate, ZigBee

\section{INTRODUCTION}

The problems related to the cardiovascular system which are generally diagnosed by electrocardiogram (ECG) and blood pressure (BP) will not give clear information about peripheral blood circulation, volume changes and oxygen contents in the blood in relation with heart functioning. With each heart beat a pulse radiates out to the peripheral circulation causing a significant change in the arterial and capillary diameters which is equivalent to the changes in volume of the blood flowing in these vessels, as shown in Fig.1. The characteristics of these pulses are unique and are influenced by arterial ageing and diseases [1]. These pulses can be detected non-invasively using PPG system. The measurement of blood volume changes in the peripheral circulation beneath the skin by means of PPG depends on the fact that blood absorbs infrared light more strongly than skin and tissues [2]. The change in blood volume caused by the pressure pulse can be detected by illuminating the skin from a light source and measuring the amount of light transmitted to a photo-detector. The signal obtained from the photo-detector is related to pulsatile arterial blood volume changes and the signal is called as PPG, which provides a means of determining the properties of the vascular tissue, peripheral blood circulation and cardiac activity during each cardiac cycle. 


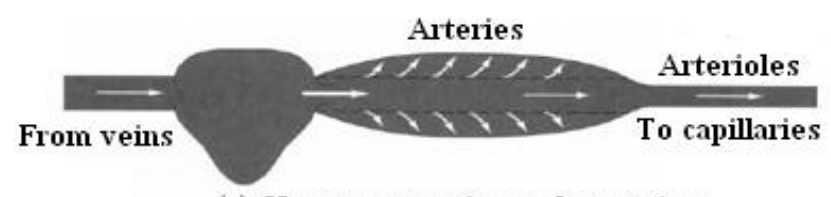

(a) Heart contracting and emptying

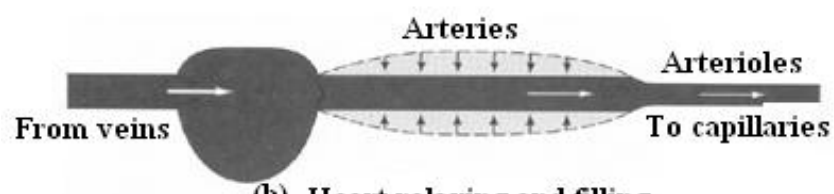

(b) Heart relaxing and filling

Fig. 1 Change in the arterial diameters with each heart beat

The PPG signal is complex and has both high frequency (pulse shape) and low frequency components (vasomotor activity) [3]. The main peripheral sites where the PPG can be obtained are the tissue pads of the fingers, ears and toes where there is a high degree of superficial vasculature [2]. The studies reveal the differences in the characteristics of PPG signals such as pulse timing, amplitude and shape features in accordance with the changes in blood volume. Thus by studying the pressure pulses the important information about the cardiovascular mechanism can be extracted.

Monitoring the peripheral pulse is more useful and dependable than monitoring the heart rate derived from the ECG in the case of a heart block because it can immediately indicate the cessation of blood circulation in the limb terminals. Moreover, a photoelectric pick-up transducer is much easier to apply than the three ECG electrodes. The amplitude of the PPG signal obtained is also quite large as compared to the ECG signal and therefore gives better signal-to-noise ratio [4]. However, the technique is severely subject to motion artifacts and need to be taken care [5].

\section{MATERIALS AND METHODS}

\subsection{PPG Signal Acquisition}

In the present work a data acquisition system (DAS) has been developed to acquire, monitor and store the PPG signal as shown in Fig. 2. The transmittance method PPG system consists of a ultra-red LED $(660 \mathrm{~nm})$ as light source and light dependent resistor (LDR) as a photo detector, amplifier, filter, ADC and PC. The LED and LDR are mounted in an enclosure in the form of a finger probe that fits over the tip of the patient's index finger with good contact to the skin surface and their optical axes are parallel to each other. Both LED and LDR are matched in their wavelength and compactness, and are highly sensitive with fast response. When the power is switched on, light is emitted by LED transmits through the fingertip of the subject's finger and the unabsorbed transmitted light is detected by the photo-detector. With each contraction of the heart, blood is forced to the extremities and the amount of blood in the finger increases [6]. It alters the optical density with the result that the light transmission through the finger reduces and the voltage in the output circuit of photo detector varies accordingly due to change in the LDR resistance. The low magnitude voltage of approximately 6 $\mathrm{mV}$ is amplified using an amplifier with a gain of 100 . A high pass filter reduces the size of the dominant DC component and enables the pulsatile AC component to be boosted to a nominal $0.6 \mathrm{~V}$ peak-to-peak level. A notch filter is used to remove $50 \mathrm{~Hz}$ power line interference that might be present in the signal and a $2^{\text {nd }}$ order Butterworth low pass filter with a cutoff frequency $8 \mathrm{~Hz}$ is used to remove any high frequency noise. The reduced noise output voltage which varies with the amount of blood in the finger and its wave shape closely follows the pressure pulse is displayed on a digital storage oscilloscope.

The PPG signal is further digitized, acquired, monitored and stored in a computer system using a dedicated DAS with built-in ADC. The DAS has 8 channels with voltage range of $\pm 10 \mathrm{~V}$ and resolution of $\pm 19.5 \mathrm{mV}$. Figure 3 shows the PPG data recording from a subject under stationary condition. The PPG signal acquired during non stationary may be affected by various noises in the patient's environment [7]. The acquired PPG pulses are further processed using Matlab signal processing tool to reduce noises introduced, if any, due to motion artifacts, ambient light interferences, and poor contact of light sources with the skin surface [8]. The moving average method, adaptive filters or periodic moving average filters can be used to remove motion artifacts [9], [10].

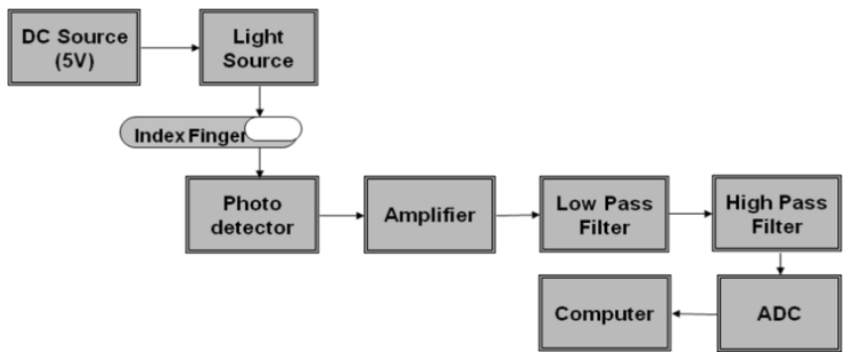

Fig. 2 Block diagram of basic PPG acquisition system

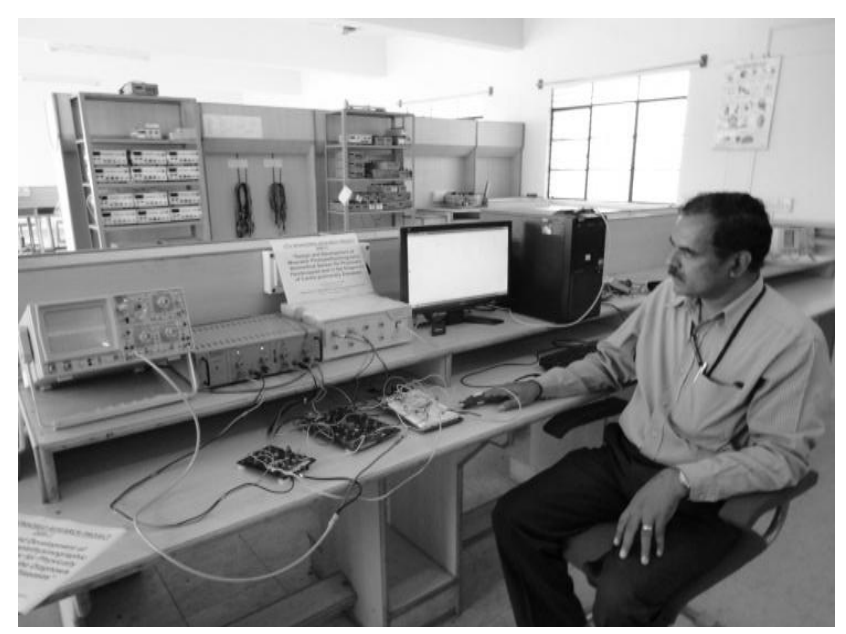

Fig. 3 Photograph of the experimental setup showing PPG signal recording from a subject

PPG works according to two physical principles. First, the pulse wave is generated based on changes in blood volume in the arteries and capillaries (Fig. 4). Second, the fact that oxyhemoglobin $\left(\mathrm{HbO}_{2}\right)$ and reduced hemoglobin $(\mathrm{Hb})$ have 
different absorption spectra. It is assumed that the amount of light absorbed by tissue and skin will remain constant irrespective of their absorbency. With this assumption it is observed that the only change in the absorption of the transmitted light will be from the increase or decrease of the blood volume in the arteries and capillaries. The measured volume change is actually an average of all of the arteries and capillaries in the space being measured. The signal that is received is dependent on the tissue type, skin type, position of the receiver and transmitter, blood volume content of the arteries and capillaries, and the properties of the sensor and receiver [6]. The absorbance of light radiation has a pulsatile component, which is due to the fluctuations in the volume of arterial blood between the source and the detector. The DC component of very low frequency corresponds to absorption due to non pulsatile arterial blood, venous and capillary blood and tissue.

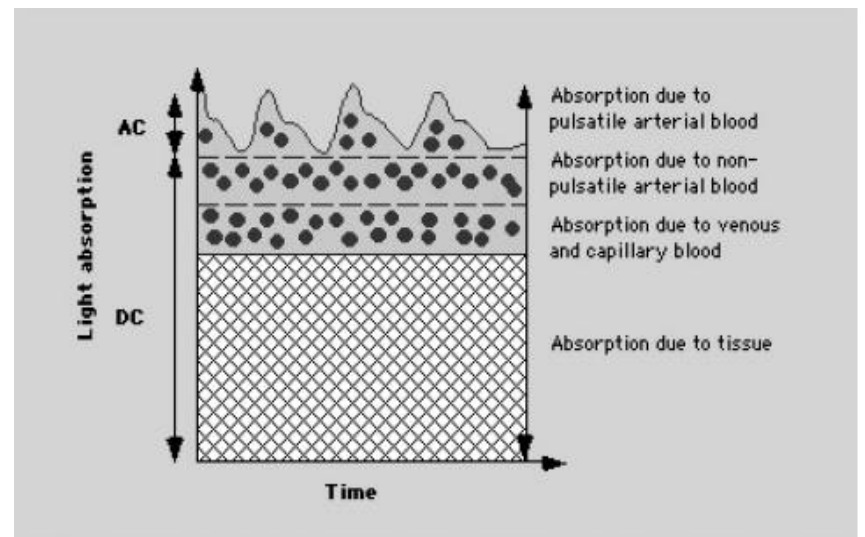

Fig4 Light absorption due to tissue, pulsatile, non pulsatile arterial blood, venous and capillary blood

\subsection{Wireless Transmission and Reception of PPG}

The current systems used by hospitals have become unsuited for continuous long duration real-time monitoring. The systems are cable based, large in size, more power consumption and not easy to carry; also require to be used next to the patient. These limitations reduce the mobility of patients and medical staff, increasing their burden and risk. Also, in order to frequently measure the patient's physiological indicators from home during normal activities (such as people with chronic diseases or elderly patients, etc.); wireless monitoring is found to be more appropriate and effective. In order to achieve this basic PPG system is further developed into a wireless PPG system using ZigBee wireless technology as shown in Fig. 5.

\subsubsection{ZigBee Wireless Module:}

The developed wireless real-time PPG health monitoring system is based on ZigBee wireless standard which is of low-cost, low power consumption and flexible network topology. In this work point to point communication between the two ZigBee modules is used. All wireless sensor hardware operates upon a short-range communication between 40 to 120 meters. The ZigBee based on IEEE 802.15.4 standard, defines the characteristics of the physical and the medium access control (MAC) layer for the low power of personal area network (PAN). The power of ZigBee module is about $2 \mathrm{~mW}$ when running operation and lower than 1 micro-watt when is in sleep operation modes [11], [12].

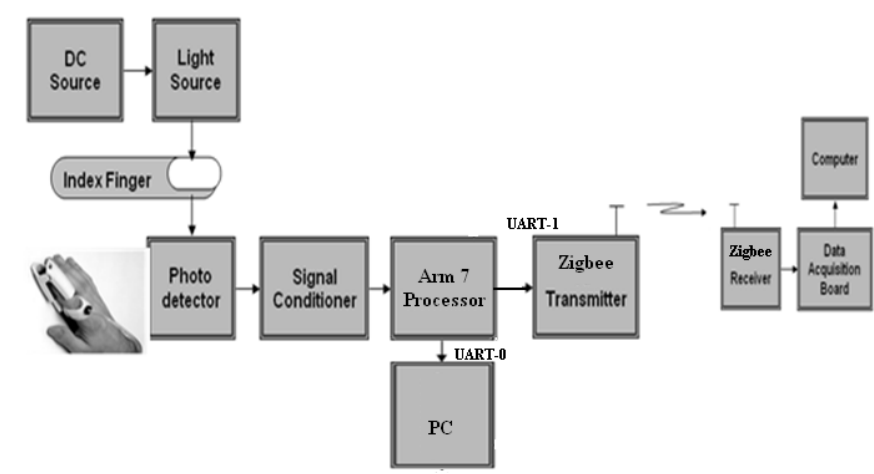

Fig5 Block diagram of PPG signal Acquisition with ZigBee transmitter and receiver

\subsubsection{PPG Transmitter Module}

Second stage of the work is the development of wireless remote monitoring of the real-time PPG data of subjects using standard ZigBee wireless technology. The transmitter section consists of PPG unit, ARM processor, personal computer and ZigBee wireless module. The acquired and signal conditioned PPG signal is received by the ARM Processor which has inbuilt ADC. ARM processor is interfaced with PC for display of PPG signals and can be analyzed using Matlab code to find its pulse rate. In this work ARM processor is used for processing the acquired PPG signal, which sends the PPG data to ZigBee transmitter module for further communication. [11], [12].

\subsubsection{PPG Receiver Module:}

The receiver section consists of ZigBee module which receives PPG data. The data received subsequently is transferred to a PC by RS232 port. The PC is used for realtime monitoring and storing the PPG data for further analysis and diagnosis. The physician can also access current PPG data and able to compute PR, APR, PRV and frequency spectrum using Matlab [11]. The professional medical staff will observe the data and do statistical analysis to provide necessary consulting services, achieving the remote medical monitoring and treatment.

In the present work PPG signals are acquired from 20 normal male subjects aged between 26 to 52 years $(41 \pm 7.1)$ and 10 female normal subjects aged between 28 to 44 years (35.2 \pm 5.4$)$ by using the above developed system at a sampling rate of 240 samples/second. The data is collected for different durations of $1 \mathrm{~min}, 2,3,4 \& 5 \mathrm{~min}$ in order to assure the reliability and repeatability of the data. All the PPG signals can be analyzed by developing algorithms in Matlab to find the PR, APR, PRV and frequency components. The local hospital ethical committee has given the consent and clearance to carry out the present work. 


\section{RESULTS AND DISCUSSION}

The pressure pulse wave changes in shape as it moves toward the periphery and undergoes amplification and alterations in its shape and temporal characteristics. These changes may be due to reflection of the pulse wave and the tapering down of the arteries towards the periphery. The shape of the pulse will significantly change during abnormalities in the blood vessels and blockages [13].

Figure 6 shows the real-time display of PPG signal on a digital CRO. Figure 7 shows the typical PPG signals acquired from a normal male subject before transmission and Figure 8 shows PPG of the same subject at receiver section located at a distance of 50 meters from transmitter. It is observed that PPG signal before and after transmission is found to be same without any distortion. The acquired PPG pulse shows two phases, the anacrotic phase being the rising edge of the pulse, and the catactrotic phase being the falling edge of the pulse. The first phase is primarily concerned with systole and second phase with diastole [14].

Figure 9 and 10 shows the PPG pulses of 1 min recorded data and the corresponding PR calculated for the subject 1 and 2 respectively. The calculated PR values are in good correlation with that of PR measured by standard pulse oximeter as shown in Table 1 for five normal subjects. The PR calculated at the transmitter and receiver side for the different durations is also in good correlation with standard method.

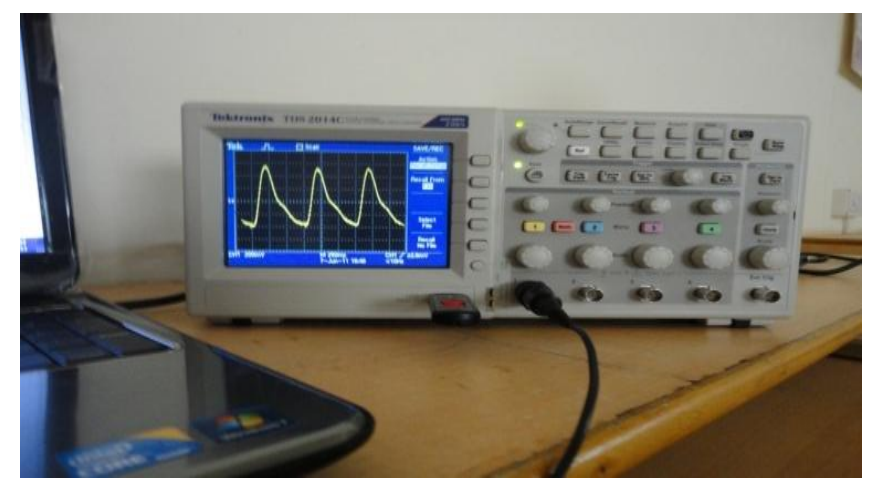

Fig6 Photograph showing the real-time display of PPG signal on digital CRO

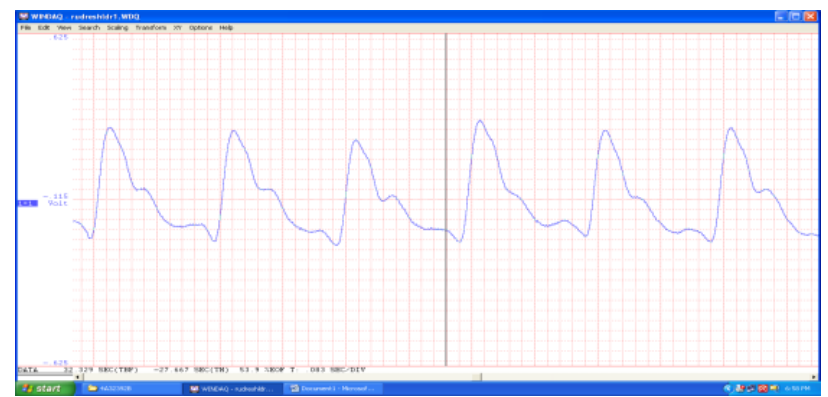

Fig7 PPG signal of a normal subject-1 at transmitter side

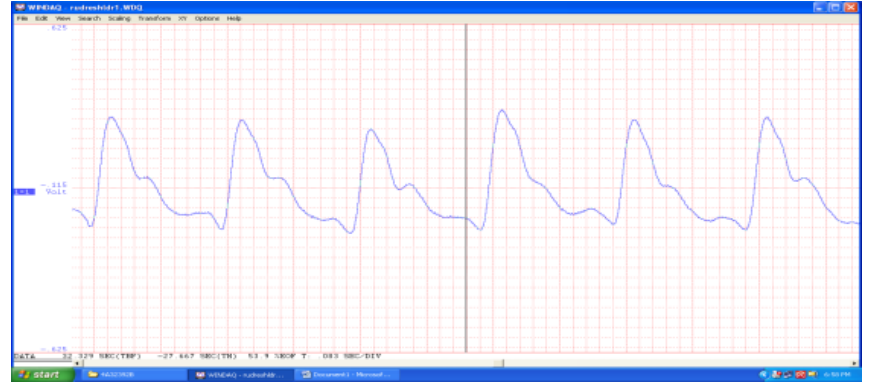

Fig8 PPG signal of a normal subject-1 at receiver side

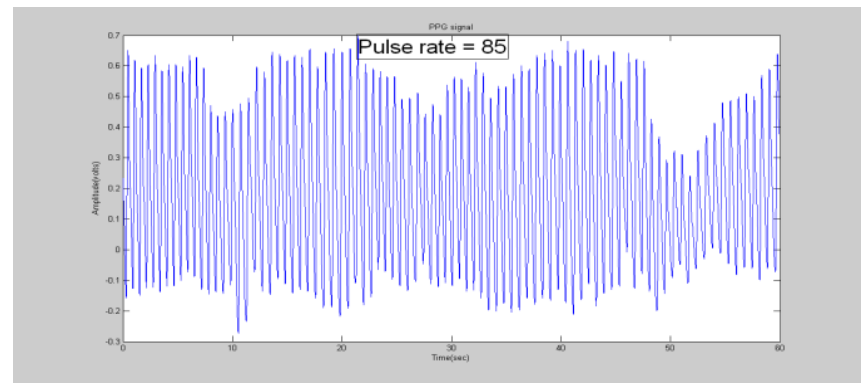

Fig9 PPG of 1 minute duration with PR (normal subject-1)

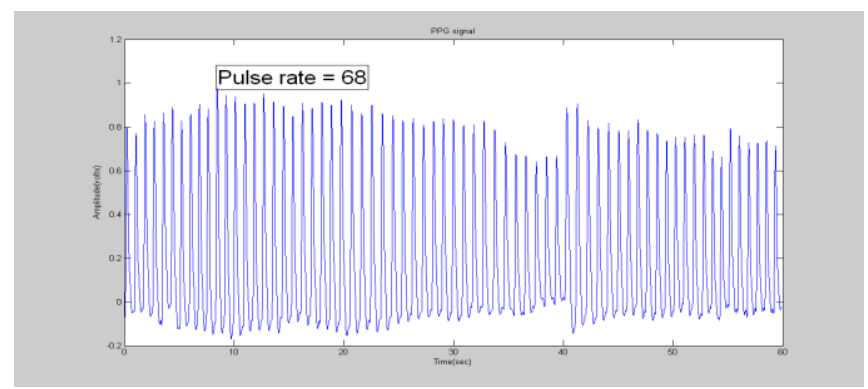

Fig10 PPG of 1 minute duration with PR (normal subject-2)

Table -1 Pulse rate (PR) recorded from normal subjects

\begin{tabular}{|l|l|l|}
\hline $\begin{array}{l}\text { Normal } \\
\text { Subject }\end{array}$ & $\begin{array}{l}\text { Pulse Rate from } \\
\text { determined from } \\
\text { developed PPG } \\
\text { system) }\end{array}$ & $\begin{array}{l}\text { Pulse Rate } \\
\text { (obtained pulse } \\
\text { standard } \\
\text { oximeter system) }\end{array}$ \\
\hline Subject -1 & 85 & 83 \\
\hline Subject -2 & 68 & 69 \\
\hline Subject -3 & 76 & 76 \\
\hline Subject -4 & 82 & 81 \\
\hline Subject -5 & 73 & 73 \\
\hline
\end{tabular}

\section{CONCLUSIONS}

In the present work an accurate and reliable electro-optical based wireless PPG data acquisition system is developed for monitoring and analysis of PPG signal. PPG signals recorded are found to be accurate and precise. The preliminary work carried out on normal subjects to find PR of cardiovascular system for different durations of PPG data is found to be accurate and is in good correlation with standard traditional methods. These results show that 
developed PPG system can be used as an alternative diagnostic tool to study the functionality of cardiovascular system and monitor the high risk heart patients remotely. The future work of this study is to find APR, PRV and frequency spectrum of PPG at both transmitted end and receiver end. To study the effect of different heart ailments on the sympathetic and the parasympathetic nervous systems and respiratory related ailments in conjunction with ECG.

\section{ACKNOWLEDGEMENTS}

The authors acknowledge the Visvesvaraya Technological University, Belgaum, for sponsoring the project under Research Promotion Scheme.

\section{REFERENCES}

[1]. Allen J. and A. Murray, "Age-related changes in the characteristics of the photoplethysmograph pulse shape at various body sites." Physiological measurement, vol.24, pp.297-307, 2003.

[2]. A.B.Hertzman, "photoelectricplethysmograph of the fingers and toes in man" Proc.Soc.Exp.Biol.Med. vol.37, pp.529-534, 1937.

[3]. Barron S. A, Rogowski Z, Kanter Y and Hemli J, "DC photoplethysmography in the evaluation of sympathetic vasomotor responses", Clin. Physiol., vol.13, pp. 561-72, 1993.

[4]. Foo J. Y. and Wilson S. J., "A computational system to optimize noise rejection in photoplethysmography signals during motion or poor perfusion states", Med. Biol. Eng. Comput., vol.44, pp. 140-145, 2006.

[5]. Rhee S, Yang B. H and Asada Hh H, "Artifactresistant power-efficient design of finger-ring plethysmographic sensors", IEEE Trans. Biomed. Eng., vol.48, pp.795-805, 2001.

[6]. Hertzman A.B and Spielman C, "Observations on the finger volume pulse recorded photoelectrically", Am. Jl. Physiol, vol.119, pp.334-335, 1937.

[7]. E Gil, M Orini, R Bail'on, J M Vergara,L Mainardi and P Laguna,"Photoplethysmography pulse rate variability as a surrogate measurement of heart rate variability during non-stationary conditions" Physiol. Meas. 31 (2010) 1271-1290

[8]. B. S. Kim and S. K. Yoo, "Motion artifact reduction in photoplethysmography using independent component analysis," IEEE Trans Biomed Eng, vol. 53, pp. 566-8, 2006.

[9]. Han-Wook Lee, Ju-Won Lee, Won-Geun Jung, and Gun-Ki Lee*The Periodic Moving Average Filter for Removing Motion Artifacts from PPG Signals" International Journal of Control, Automation, and Systems, vol. 5, no. 6, pp. 701-706, December 2007.

[10]. Lee, W. Jung, I. Kang, Y. Kim, and G. Lee, "Design of filter to reject motion artifact of pulse oximetry," Comput. Stand. Interfaces, vol. 26, no. 3, pp. 241249. 2004

[11]. N.Watthanawisuth, T. Lomas, A. Wisitsoraat, A.Tuantranont, "Wireless Wearable Pulse Oximeter for Health Monitoring using ZigBee Wireless Sensor Network", Nanoelectronics and MEMS Laboratory,
National Electronics and Computer Technology Center(NECTEC)., Pathumthani, Thailand 12120.

[12]. Wang Feng Qin, Li.Yang "ZigBee Technology for Designing and Implementing a Remote Medical Monitoring System", International Conference on Computer, Mechatronics, Control and Electronic Engineering (CMCE), pp. 172-175, 2010.

[13]. O'Rourke M F and Gallagher D E 1996 Pulse wave analysis J. Hypertens. Suppl. 14 S147-57.

[14]. Allen J, "Photoplethysmography and its application in clinical physiological measurement", Physiol. Meas., vol.28, R1-R39, 2007.

\section{BIOGRAPHIES}

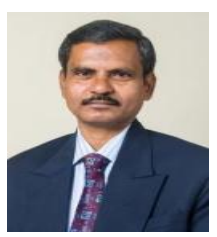

Prof. Jayadevappa B.M received the M.E degree in Electronics and Control from Birla Institute of Technology and Science, Pilani, Rajasthan, India, in the year 1994. He has 27 years of teaching and 05 years of research experience. His Research interests are Biomedical Signal Processing and Rehabilitation Engineering. Currently, he is Professor and Head, Department of Electronics and Instrumentation Engineering, Bapuji Institute of Engineering and Technology, Davangere, Karnataka, India.

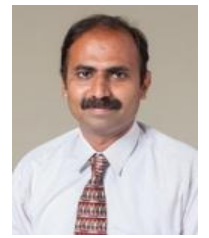

Mr.Kiran Kumar G.H received the M.Tech. degree in Digital Electronics from BVBCET, Hubli, Karnataka, India, in the year 2003. He has 11 years of teaching and 01 year of research experience. His Research interests are Biomedical Signal Processing and wireless sensor network. Currently, he is Assistant Professor in the Department of Electronics and communication Engineering, Bapuji Institute of Engineering and Technology, Davangere, Karnataka, India.

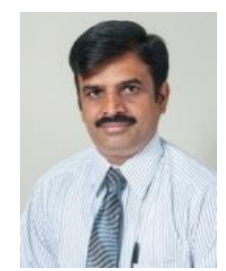

Mr.Anjaneya L.H received the M.Tech. degree in Biomedical Engineering from MIT, Manipal, Karnataka, India, in the year 2000. He has 15 years of teaching and 05 year of research experience. His Research interests are Biomedical Signal Processing and Rehabilitation Engineering. Currently, he is Associate Professor in the Department of Biomedical Engineering, Bapuji Institute of Engineering and Technology, Davangere, Karnataka, India.

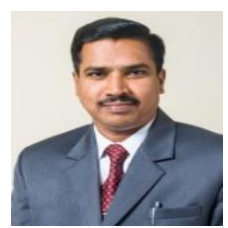

Dr. Mallikarjun S. Holi, received the Ph.D. degree in Biomedical Engineering from Indian Institute of Technology, Madras, Chennai, India, in the year 2004. He has 20 years of teaching and 12 years of research experience. His Research interests are Biomechanics, Biomedical Signal and Image processing, and Rehabilitation Engineering. Awarded Institute Merit Prize for the Best Academic record in M.Tech. (Biomedical Engineering) program for the academic year 1995-1996 from Indian Institute of 
Technology, Madras. Recipient of Travel Award from IEEE-EMBS in 2001 and from IBMS \& JSBMR in the year 2003. Currently, he is Professor and Chairman, Department of Electronics and Instrumentation Engineering, University B.D.T. College of Engineering, Davangere, Karnataka, India. 